\section{The human sense of smell}

\section{Elena Cantone, ${ }^{1}$ Filippo Ricciardiello, ${ }^{2}$ Rossella Cuofano, ${ }^{1}$ Giovanni Castagna, ${ }^{1}$ Flavia Oliva, ${ }^{2}$ Giulio Sequino, ${ }^{2}$ \\ Teresa Abate, ${ }^{2}$ Romolo Villani, ${ }^{3}$ \\ Maurizio Iengo ${ }^{1}$}

${ }^{1}$ Department of Neurosciences, ENT Section, "Federico II" University of Naples; ${ }^{2}$ Otorhinolaryngology Unit, Cardarelli Hospital, Naples; ${ }^{3}$ Department of Anesthesiology, Cardarelli Hospital, Naples, Italy

\begin{abstract}
The sense of smell, like taste and trigeminal senses, is a chemical sense dedicated to the perception of chemical stimulations and to the generation of responses to them. Although, from an evolutionary perspective, the chemical senses are the oldest of our senses, our knowledge on the neural processing of the three chemical senses is still incomplete and has been considerably lagging behind that of our other senses. The current review aims to give an overview about human smell function. In particular we focus on the anatomy and physiology of the olfactory system, the epidemiology and the causes of olfactory loss, and on the clinical management of olfactory disorders stressing the impact of smell loss on the quality of life. Lastly, we emphasize the importance of olfaction in every day life. In our opinion, the impairment of sense of smell should be taken into serious consideration by the clinicians, as it could be the indicator of important systemic diseases or the cause of domestic accidents.
\end{abstract}

\section{Introduction}

The sense of smell is of utmost importance for human quality of life, health, and survival. Indeed, the role of olfaction is to guide our attention towards hazards (e.g., microbial threats and poisonous fumes) or, conversely, towards items with positive connotations (e.g., nutritious food). ${ }^{1}$

This guidance is predominantly driven by the ecological valence (pleasantness/ unpleasantness) of the odorous items (e.g., food), which, to a large part, is determined by the individual's personal history with that item. ${ }^{1}$ The relatively strong positive or negative emotions often elicited by smells are also shaped by prior experience, and believed to enhance the appropriate behav- ioral response. ${ }^{2}$ Indeed, the sense of smell is involved in social communication. ${ }^{2}$ Furthermore, there is evidence that odors elicit emotion and are linked to emotional memory. ${ }^{3}$ Nowadays, thanks to neuroimaging studies, we know that the amygdala, orbitofrontal cortex (OFC) and hippocampus are involved in the process of odorelicited emotion and odor-associated emotional memory. ${ }^{3}$

\section{A brief overview of the anatomy and physiology of the olfactory system}

Smell perception depends on the olfactory receptor neurons (ORNs), highly specialized cells of the olfactory epithelium (OE), able to perceive the identity and concentration of odors. ORNs are bipolar cells with a dendritic end containing 3-50 cilia that project into overlaying mucus; their unmyelinated axons form bundles projecting to the cribriform plate and synapse in the olfactory bulb (OB). ${ }^{4}$ The main feature of the 6-30 million ORNs is that they continuously renew themselves from the basal cells. ${ }^{5}$ This regeneration capacity diminishes with increasing age. ${ }^{5}$ Two other cell types, basal cells and supporting cells, form the OE. This entire apparatus - mucus layer and epithelium with neural and supporting cells - is called olfactory mucosa. ${ }^{6}$ The olfactory mucosa is localized in the upper third of the nasal cavity, extending for about $5 \mathrm{~cm}^{2}$; only about $10 \%$ of inhaled air reaches the OE. ${ }^{5}$ Odoriferous substances penetrate the mucous membrane and are absorbed into the mucus covering the OE, largely derived from specialized Bowman's glands. In these secretions the odorant-binding proteins favor the transit of hydrophobic odorants to the olfactory receptors. ${ }^{7}$ Subsequently, odors activate the olfactory receptor (ORs) proteins located in the cilia of ORNs. ${ }^{7}$ Over 350 different functional receptor proteins are expressed in the cilia of human ORNs. ${ }^{7}$ Every ORN expresses a certain receptor type. ORNs that express the same receptor type send axons to specific glomeruli in the OB. Odors regularly bind to more than one type of receptor. ${ }^{5}$ This leads to various patterns of stimulation in the $\mathrm{OB}$ that serve to code the quality of odors. ${ }^{5}$ The ORs are members of the heptahelical G-protein-coupled receptor (GPCR) superfamily whose genes are distributed on chromosome 11, 1, 6 , and 9. Odorants bind to receptor pockets located on receptor transmembrane domains. The transduction results from the activation of a GTP-binding protein, which
Correspondence: Elena Cantone, Department of Neurosciences, ENT Section, "Federico II" University of Naples, Via Pansini 5, 80100 Naples, Italy

Tel. +39817463598 - Fax +39817463592 .

Email: elena.cantone@unina.it

Key words: Olfaction; Smell; Disorders; Dysosmia; Sniffin'sticks test.

Received for publication: 17 January 2017.

Revision received: 4 March 2017.

Accepted for publication: 9 March 2017.

This work is licensed under a Creative Commons Attribution 4.0 License (by-nc 4.0).

(C) Copyright E. Cantone et al., 2017

Licensee PAGEPress, Italy

Translational Medicine Reports 2017; 1:6579 doi:10.4081/tmr.6579

activates type III adenylyl cyclase, catalyzes the production of 3,5-cyclicmonophosphate (cAMP) and opens the cyclicnucleotide-gated channels. This results in the cellular influx of sodium and calcium ions with consequent depolarization of the cell. Further amplification occurs from the opening of calcium-activated chloride channels and the resultant efflux of $\mathrm{Cl}^{-}$ from the cell. ${ }^{4,7,8}$ The ORNs are glutamatergic and provide excitatory input to the OB. ${ }^{4}$ Axons from ORNs expressing the same OR coalesce and synapse with dendrites of second-order projection neurons, called mitral and tufted cells (MTc) forming a structure called glomerulus. ${ }^{4}$ While each glomerulus receives input for ORNs expressing the same OR, there is no chemotropic map in the OB. ${ }^{4}$ The glomerular cells (GCs) are inhibitory neurons with the function of inhibition of MT lateral dendrites contributing to the processing of olfactory discrimination. ${ }^{9}$ The MT cells are the main output from the $\mathrm{OB}$ to olfactory cortex, ${ }^{9}$ that includes the anterior olfactory nucleus $(\mathrm{AON})$, tenia tecta, olfactory tubercle, cortical nucleus of the amygdala, anterior and posterior piriform cortex (PC), and the lateral entorhinal cortex. ${ }^{10}$ In addition, olfaction includes a thalamo-cortical component. ${ }^{10}$ The mediodorsal nucleus of the thalamus (MDT) receives olfactory input from the olfactory cortex and projects in turn to the orbitofrontal cortex (OFC). The OFC also has direct, reciprocal connections with the anterior PC. The OFC is a highly multisensory region important for information regarding reward value and decision making. ${ }^{10,11}$ In summary, each cortical region has a specific function: autonomic regulation and emotional processing (amygdala) ${ }^{12}$ to motivated behavior (olfac- 
tory tubercle), to episodic and working memory (entorhinal cortex - hippocampus), to reward valuation and decision making (OFC), ${ }^{13}$ and to more basic odor quality discrimination (PC). ${ }^{10,11}$ So far, we have considered the main olfactory epithelium (MOE), which projects to the main olfactory bulb (MOB). In addition, in some animal species, there is an accessory olfactory system with its peripheral receptors located in the vomeronasal organ (VNO) which projects to the accessory olfactory bulb (AOB). The VNO seems to contribute to the detection of pheromones. The VNOs of humans and some primates are non-functional, although, according to some authors, pheromones play important roles in eliciting social and reproductive behavior, and in humans, the MOB may be responsible for pheromone detection. ${ }^{11}$ The olfactory system is unique among the senses as it projects initially to cortical regions, rather than thalamic nuclei. Thalamic connections occur post-cortically, along with projections from $\mathrm{PC}$ to other limbic regions, such as the hypothalamus and hippocampus. Projections to the OFC can be either direct from primary olfactory cortex, or indirect via the dorsomedial nucleus of the thalamus. In addition, in the olfactory system the signals from primary sensory receptors to higher processing regions are predominantly sent ipsilaterally, rather than having a contralateral representation as the other senses. This fact allows the assessment of the relative contribution of each hemisphere to the processing of olfactory stimuli. If stimulus presentation is restricted to one nostril only, the ipsilateral hemisphere would be preferentially and initially engaged. ${ }^{14}$

\section{Smell loss: epidemiology}

The prevalence of olfactory dysfunction is about $3.8 \%$ in adults between 21 and 84 years of age, and tends to increase with age (from $0.6 \%$ in those $<35$ years to $13.9 \%$ among those $\geq 65$ years), with higher prevalence in men. ${ }^{14}$ Half of the elderly population between 65 and 80 years have evident olfactory dysfunction. ${ }^{14}$ The under-reporting of smell loss is likely to be related to unawareness of smell loss, which is common in the elderly. ${ }^{2}$ Factors involved in agerelated olfactory dysfunction include changes in non-olfactory elements of the nose (e.g., airflow patterns and mucous composition), OE, OB, olfactory cortex, and neurochemical changes in the brain. ${ }^{15}$ The commonest reported etiologies of smell loss are post-viral upper respiratory tract infections (URTI) (18 to $45 \%$ of the clinical population) and nasal or sinus disease ( 7 to $56 \%$ ), followed by head traumas ( 8 to $20 \%$ ), exposure to toxins or drugs ( 2 to $6 \%$ ), and congenital loss ( 0 to $4 \%$ ). ${ }^{1,2}$ Conversely, the percentage of patients with these conditions who have clinically proven smell loss is rather high: 76 to $95 \%$ in post-viral URTI, 72 to $98 \%$ in nasal/sinus disease, 86 to $94 \%$ in head trauma, $67 \%$ in exposure to toxins/drugs, and $100 \%$ in congenital cases. ${ }^{2}$ Moreover, olfactory dysfunction has a considerable prevalence in dementia, with estimated numbers as high as $100 \%$ in Alzheimer's disease, 90\% in Parkinson's disease; $96 \%$ in the frontal variant of Frontotemporal dementia and $15 \%$ in vascular dementia. ${ }^{15}$
Smell loss: impact on quality of life

Smell loss may have adverse effects on general QoL and can lead to depression, feelings of vulnerability, altered food intake, reduced social interaction, and decreased intimacy with a partner. ${ }^{1,2}$ Smell loss is generally accompanied by a slight reduction in the ability to taste, probably due to the lack of central nervous system interactions between the chemical senses. ${ }^{16}$

Furthermore, decreased olfactory functions significantly influence the everyday safety. Indeed, almost all patients complained of difficulties with cooking, of eating spoiled food, of too little perception of their own body odor, of burning food, and of problems at work. In addition to this, a large number of patients suffering from olfactory impairment are at risk of toxic exposures and may be unable to detect leaking gas, smoke, or the odor of rotting food. This might explain why patients with olfactory disorders, particularly older people, are more likely to encounter hazardous events (e.g., burning food, ingesting spoiled food, fire) than normosmic. These hazards have to be especially mentioned when counseling patients with smell loss.

A limitation in the studies on QoL is the heterogeneity of studied groups with respect to either the type of smell disorder (sensitivity loss, smell distortion, or phantom smell) or the primary etiology underlying the disorder, making it difficult to conclude to what extent the impact on QoL can be referred to a specific type of disorder or etiology.

Table 1. Quantitative and qualitative dysosmias.

\begin{tabular}{|c|c|c|c|}
\hline & Quantitative disorders & Qualitative disor & \\
\hline Disorder & Characteristics & Disorder & Characteristics \\
\hline Anosmia & Total loss of olfactory function & Olfactory intolerance & $\begin{array}{l}\text { Subjective hypersensitivity } \\
\text { toward odorants, but normal olfactory function }\end{array}$ \\
\hline Functional anosmia & $\begin{array}{l}\text { Significantly impaired olfaction, } \\
\text { including both total loss and minimal } \\
\text { residual perception }\end{array}$ & Parosmia* (synonym, troposmia) & $\begin{array}{l}\text { Abnormal perception, with sensory input } \\
\text { present }\end{array}$ \\
\hline Partial anosmia & $\begin{array}{l}\text { Significantly reduced sensibility toward } \\
\text { one odorant or group of odorants, } \\
\text { compared with the general population } \\
\text { without clinical relevance }\end{array}$ & Phantosmia* & Abnormal perception, with sensory input absent \\
\hline Hyposmia & Partial loss (frequent, e.g., in old age) & Olfactory agnosia & $\begin{array}{l}\text { Inability to recognize the olfactory } \\
\text { sensation despite the olfactory processing } \\
\text { functions and intellectual are intact }\end{array}$ \\
\hline Hyperosmia & $\begin{array}{l}\text { Hypernormal olfactory sensitivity } \\
\text { (very rare, e.g., in migraine) }\end{array}$ & - & - \\
\hline
\end{tabular}

*Diagnoses of parosmia and phantosmia are based solely on the self-report of the patient. 


\section{Classification and causes of smell disorders}

Dysosmias are transient or permanent alterations of smell. We, traditionally, use different types of classifications: the commonest distinguishes quantitative and qualitative forms, as in the diagram below (Table 1). The etiological classification, distinguishes idiopathic, congenital and acquired forms. The acquired forms are classified, in turn, as conductive or sensorineural forms.

Conductive forms are secondary to mechanic and obstructive causes (high deviations of the nasal septum, trauma with subsequently deformation of the nasal pyramid, surgery), inflammation of the nasal mucosa (acute and chronic rhinosinusitis, with and without nasal polyposis) and defects of breathing (following a tracheotomy or surgical removal of the larynx, congenital anomalies, etc.) with integrity of the respiratory and olfactory centers. Sensorineural forms are secondary to lesions of the neuroepithelium or olfactory centers (tumors, degenerative processes such as multiple sclerosis and toxic/metabolic toxic or metabolic polyneuritis - e.g. diabetes, age, viral infections poisoning by salts of chromium, lead, mercury, tobacco abuse, vasoconstrictors, morphine, cocaine, etc.).
The main etiologies of olfactory dysfunction are listed in the chart below (Table 2). ${ }^{17}$

\section{Clinical and instrumental assess- ment of smell disorders}

The medical history of the patient suffering from olfactory dysfunction must be accurately collected by the clinicians. ENT evaluation and nasal endoscopy is mandatory to exclude sinonasal diseases. It might be also required a complete neurological evaluation of the cranial nerves, cerebellar function and sensorimotor function. When a psychiatric etiology is suspected, it is recommended to send the patient to qualified specialists. Standardized questionnaires are useful to assess patients with smell and taste disorders. ${ }^{2}$ For instance, validated psychophysical tests (e.g., tests of odor detection, identification, discrimination, memory, and suprathreshold intensity), and electrophysiological tests (e.g., odor event-related potentials) are of utmost importance to confirm the clinical suspicion of the olfactory impairment.

Psychophysical tests require a conscious response by the patient. Despite the fact that a number of clinical olfactory tests (about 200) $)^{2}$ have been described in the literature, only a few have achieved widespread acceptance and are available commercially. ${ }^{18}$ The most widely used of these is the 40-item University of Pennsylvania Smell Identification Test (UPSIT). UPSIT has been administered to nearly 500,000 patients. ${ }^{19}$ UPSIT comprises four booklets, each containing 10 microencapsulated (scratch and sniff) odors released by the scraping of a pencil tip. The examinee must provide an answer on each test item even if no odor is perceived or the perceived odor does not smell like one of the proposed alternatives (i.e., the test is forced-choice), its main limitation is that some odorants are not universally recognized because they are familiar to North American population but not to European populations. $^{20}$

Odor threshold tests are conceptually analogous to pure-tone hearing threshold tests, except that the stimuli consist of a range of concentrations of an odorant, rather than a range of tones. In a given test, a series of different concentrations of an odorant are presented to a subject via sniff bottles, squeeze bottles, felt-tip pens, or olfactometers, such as the one depicted in. The goal of the test is to detect the lowest odorant concentration that can be reliably detected. Among these, Sniffin' Sticks (SS Burghart Medical Technology, Wesdel, Germany) is a test of nasal chemosensory function that is based on pen-like odor dispensing devices, introduced some 10 years ago by Kobal and co-workers. ${ }^{21}$ It

Table 2. Main etiologies of smell disorders listed in order of prevalence.

\begin{tabular}{|c|c|c|}
\hline & Cause & Leading etiologies \\
\hline Most common & Sinonasal conditions & $\begin{array}{l}\text { Upper respiratory infection (especially viral), allergic rhinitis, chronic rhinosinusitis, } \\
\text { nasal polyps }\end{array}$ \\
\hline More common & $\begin{array}{l}\text { Head trauma } \\
\text { Neurodegenerative disorders }\end{array}$ & $\begin{array}{l}\text { Damage to cribriform plate, shearing forces, and intracranial damage; facial trauma } \\
\text { Parkinson disease, parkinsonism, Alzheimer disease, mild cognitive impairment, multiple sclerosis }\end{array}$ \\
\hline \multirow[t]{12}{*}{ Less common } & Medications & $\begin{array}{l}\text { Chemotherapy, angiotensin-converting enzyme inhibitors, angiotensin receptor blockers, } \\
\text { dihydropyridine calcium channel blockers, diuretics, intranasal zinc, antimicrobials } \\
\text { (macrolides, terbinafine [Lamisil], fluoroquinolones, protease inhibitors, griseofulvin, penicillins, } \\
\text { tetracyclines, nitroimidazoles [metronidazole (Flagyl)]), antiarrhythmics, antithyroid agents, } \\
\text { antidepressants, anticonvulsants, lipid-lowering agents }\end{array}$ \\
\hline & Intoxicants or illicit substances & Alcohol, cocaine \\
\hline & Toxins & $\begin{array}{l}\text { Ammonia, hairdressing chemicals, gasoline, formaldehyde, paint solvents, welding agents, benzene, } \\
\text { sulfuric acids, cadmium, acrylates, iron, lead, chromium }\end{array}$ \\
\hline & Chronic medical conditions & Renal or hepatic failure, complicated type 2 diabetes mellitus, cancer, human immunodeficiency virus \\
\hline & Structural or mechanical conditions & Ischemic stroke, subarachnoid or intracranial hemorrhage, brain or sinonasal tumor \\
\hline & Nutritional deficiencies & $\begin{array}{l}\text { Malnutrition, pernicious anemia or vitamin B12 deficiency, deficiencies in vitamins B6 or A, } \\
\text { niacin, zinc, or copper }\end{array}$ \\
\hline & Postsurgical state & Nasal surgery (septal or sinus), total laryngectomy, pharyngectomy, tonsillectomy \\
\hline & Post-radiation & Especially to head and neck \\
\hline & Congenital conditions & Kallmann syndrome, anosmia \\
\hline & Psychiatric conditions & Anorexia nervosa (not bulimia), major depressive disorder, bipolar disorder, schizophrenia \\
\hline & Endocrine conditions & Pregnancy, hypothyroidism, Addison disease, Cushing syndrome \\
\hline & Autoimmune/inflammatory conditions & Sjögren syndrome, systemic lupus erythematosus, sarcoidosis, herpes encephalitis \\
\hline
\end{tabular}


consists of tests for odor threshold, discrimination, and identification, with the latter two being supra-threshold tests. The threshold test indicates the concentration at which the odor is reliably detected. The odorants most frequently used are n-butanol or phenylethyl alcohol, because of their minimal trigeminal components. The discrimination test tests the ability of distinguishing odors. The identification test is similar to the University of Pennsylvania Smell Identification Test (UPSIT). Previous work established its test-retest reliability and validity. Like UPSIT is a forced-choice test. Results of the test are presented as the sum of results obtained for threshold, discrimination, and identification measurements, the TDI score. ${ }^{22}$ Functional anosmia is diagnosed when TDI score is less than $16 .^{23}$

SS is widely validated in Europe ${ }^{24}$ and its advantage is that allows a more detailed assessment of smell and that it is reusable over UPSIT. ${ }^{25}$

Electrophysiological tests do not require patient cooperation and are primarily used for scientific research and medico-legal purposes. A practical electrophysiological procedure is the measurement of odor-induced electrical activity at the level of the scalp (e.g., the odor event-related potential or OERP). This activity reflects odor-related changes induced in electrical fields generated by large populations of cortical neurons. ${ }^{7}$ However, the signals are small $(<50 \mu \mathrm{V})$, can be difficult extract from the background EEG, and require complex stimulus presentation equipment. In one study, for example, OERPs were not identifiable in nearly a third of subjects with no olfactory deficits. ${ }^{7}$ Besides determining OERPs, it is also possible to determine peripheral mucous membrane potentials in humans (so-called electro-olfactograms) directly from the olfactory regions. However, due to a relatively high interindividual variability of EOG responses, the current clinical value in medico-legal cases or individual patients is limited.

Structural magnetic resonance imaginc (MRI) and $\mathrm{OB}$ volume A computer tomography/magnetic resonance (CT/MRI) is recommended to exclude sinonasal or intracranial anomalies. To date, we know that $\mathrm{OB}$ volume correlates with the degree of impairment of smell. Therefore, the OB volume is considered a possible tool to assess the individual prognosis of olfactory disorders. The volumetric evaluation of $\mathrm{OB}$ can be performed on images of structural MRI. ${ }^{26}$

\section{Prognosis and treatment of smell disorders}

Recent studies suggest that the prognosis of smell disorders are related to the etiology, but larger studies have shown that prognosis is related to the severity of the loss. ${ }^{27}$ Negative prognostic factors include more severe loss of smell, longer duration of symptoms, advanced age at onset (especially patients older than 75 years), and smoking. Women may have a better prognosis than men. ${ }^{28}$ Age-related and congenital smell disorders cannot be treated successfully. ${ }^{29}$ In 10 to $20 \%$ of cases of post-traumatic anosmias there is partial remission over the years, whereas the recovery rate is much better for patients with post-viral olfactory disorders $(60 \%){ }^{29}$ The treatment of smell disorders is based primarily on the determination of the cause of the dysfunction. However, there are only limited treatments available for smell disorders. A proven effective treatment is only available where sinonasal illnesses cause the smell disorder and consist on the therapy for the underlying disease. ${ }^{29}$ Systemic steroids can be administered in idiopathic forms, starting with a dose of 40 $\mathrm{mg}$ and then reducing it every second day by $5 \mathrm{mg}$. This procedure can be used in order to rule out an inflammatory smell disorder, which is not always macroscopically visible. A certain percentage of these patients respond with an improvement in their ability to smell. ${ }^{30}$ Twelve weeks of olfactory training has been shown to increase olfactory sensitivity in one-third of patients with olfactory loss secondary to post-traumatic, post-viral, or idiopathic causes. This at-home technique involves twice-daily exposure to four odors (phenylethyl alcohol, eucalyptus, citronellal, and eugenol). ${ }^{31,32}$ This training seems to improve the olfactory performance in about $25 \%$ of patients. ${ }^{29}$

Eventually, the clinician should suggest some important rules of conduct: to use of smoke detectors in the living environment; to take precautions to avoid intake of spoiled foods; to use substances that increase the taste and hedonistic experience of food, such as spices, herbs and other additives. More can be speculated about the future. Given the plasticity of the olfactory system it could be hypothesized the autologous replanting of previously removed and amplified olfactory cells appears possible, as does the local use of growth factors and the use of electronic sensors and their attachment to the olfactory bulb.

\section{Conclusions}

Smell is a chemical warning system guiding the food intake and the social relationships. Smell disorders are considered reliable biomarkers in many sinonasal and non-sinonasal diseases as well as an early biomarker for neurodegeneration or endocrinologic diseases. ${ }^{32-35}$

Furthermore, smell disorders can markedly affect the QoL.

To determine appropriate treatment and provide adequate counseling regarding prognosis, the disorder must be accurately classified. A thorough history-including exact time course of the observed disorder-and standardized testing are mandatory. Additional investigations might be necessary before the disorder can be classified. Only then can therapy be chosen and adequate counseling be provided.

\section{References}

1. Croy I, Nordin S, Hummel T. Olfactory disorders and quality of life - an updated review. Chem. Senses 2014,39:18594.

2. Welge-Luessen A, Hummel T. management of smell and taste disorders: a practical guide for clinicians. Stuttgart: Thieme Publishers; 2014.

3. Kadohisa M. Effects of odor on emotion, with implications. Front Syst Neurosci 2013;7:66.

4. Benarroch EE. Olfactory system: functional organization and involvement in neurodegenerative disease. Neurology 2010;75:1104-9.

5. Hüttenbrink KB, Hummel T, Berg D, et al. Olfactory dysfunction: common in later life and early warning of neurodegenerative disease. Dtsch Arztebl Int 2013;110:1-7.

6. Purves D, Augustine GJ, Fitzpatrick D, et al. Neuroscience, 2nd edition. Sunderland, MA: Sinauer Associates; 2001.

7. Richard L. Doty and Vidyulata Kamath. The influences of age on olfaction: a review. Frontiers Psychol 2015;5:20-4.

8. Dorota Latek D, Modzelewska A, Trzaskowski B, et al. G protein-coupled receptors - recent advances. Acta Biochim Pol 2012;59:515-29.

9. Nuñez-Parra A, Li A, Restrepo D. Coding odor identity and odor value in awake rodents. Prog Brain Res 2014;208:205-22.

10. Wilson DA, Xu W, Sadrian B, et al. Cortical odor processing in health and disease. Prog Brain Res 2014;208:275-305. 
11. Kadohisa M Effects of odor on emotion, with implications. Front Syst Neurosci 2013;7:66.

12. Li W. Learning to smell danger: acquired associative representation of threat in the olfactory cortex. Frontiers Behav Neurosci 2014.

13. Thierry Thomas-Danguin, Charlotte Sinding, Sébastien Romagny, et al. The perception of odor objects in everyday life: a review on the processing of odor mixtures. Frontiers Psychol 2014;8:98.

14. Good KP, Sullivan RL. Olfactory function in psychotic disorders: Insights from neuroimaging studies. World J Psychiatr 2015;5:210-21.

15. Alves J, Petrosyan A, Magalhães R. Olfactory dysfunction in dementia. World J Clin Cases 2014;2:661-7.

16. Deems DA, Doty RL, Settle RG, Moore-Gillon V. Smell and taste disorders: a study of 750 patients from the University of Pennsylvania Smell and Taste Center. Arch Otorhinolaryngol Head Neck Surg 1991;117:519-28.

17. Malaty J, Malaty IA. Smell and taste disorders in primary care. Am Fam Physician 2013;88:852-9.

18. Jiang RS, Kuo LT, Wu SH, et al. Validation of the applicability of the traditional Chinese version of the University of Pennsylvania Smell Identification Test in patients with chronic rhinosinusitis. Allergy Rhinol (Providence) 2014;5:28-35.

19. Doty RL. Office procedures for quantitative assessment of olfactory function. Am J Rhinol 2001;21:460-73.
20. Doty RL, Shaman P, Dann M. Development of the University of Pennsylvania Smell Identification Test: a standardized microencapsulated test of olfactory function. Physiol Behav 1984;32:489-502.

21. Hummel T, Kobal G, Gudziol H, Mackay-Sim A. Normative data for the "Sniffin' Sticks" including tests of odor identification, odor discrimination, and olfactory thresholds: an upgrade based on a group of more than 3000 subjects. Rhinology 2007;264:237-43.

22. Lotsch J, Hummel T. The clinical significance of electrophysiological measures of olfactory function. Behav Brain Res 2006;170:78-83.

23. Gudziol V, Lötsch J, Hähner A, et al. Clinical significance of results from olfactory testing. Laryngoscope 2006;116:1858-63.

24. Haehner A, Mayer AM, Landis BN, et al. High test-re-test reliability of the extended version of the "Sniffin' Sticks" test. Chem Senses 2009;34:705-11.

25. Hummel T, Sekinger B, Wolf SR, et al. 'Sniffin' sticks': olfactory performance assessed by the combined testing of odor identification, odor discrimination and olfactory threshold. Chem Senses 1997;22:39-52.

26. Altinayar S, Oner S, Can S, et al. Olfactory disfunction and its relation olfactory bulb volume in Parkinson's disease. Eur Rev Med Pharmacol Sci 2014; 18:3659-64.

27. London B, Nabet B, Fisher AR, et al.
Predictors of prognosis in patients with olfactory disturbance. Ann Neurol 2008;63:159-66.

28. Hummel T, Lötsch J. Prognostic factors of olfactory dysfunction. Arch Otolaryngol Head Neck Surg 2010;136:347-51.

29. Hummel T, Landis BN. Smell and taste disorders. GMS Current Topics in Otorhinolaryngology - Head and Neck Surgery. Head and Neck Surgery 2011;10:1865-2011.

30. Heilmann S, Hüttenbrink KB, Hummel T. Local and systemic administration of corticosteroids in the treatment of olfactory loss. Am J Rhinol 2004;18:29-33.

31. Hummel T, Rissom K, Reden J, et al. Effects of olfactory training in patients with olfactory loss. Laryngoscope 2009;119:496-9.

32. Haehner A, Tosch C, Wolz M, et al. Olfactory training in patients with Parkinson's disease. PLoS One 2013;8:e61680.

33. Tonacci A, Billeci L, Tartarisco G, et al. Olfaction in autism spectrum disorders: a systematic review. Child Neuropsychol 2015;2015:1-25.

34. Cantone E, Maione N, Di Rubbo V, et al. Olfactory performance after crenotherapy in chronic rhinosinusitis in the elderly. Laryngoscope 2015;125:1529-34.

35. Ottaviano G, Cantone E, D'Errico A, et al. Sniffin' Sticks and olfactory system imaging in patients with Kallmann syndrome. Int Forum Allergy Rhinol 2015;5:855-61. 